\title{
PROM and Labour Effects on Urinary Metabolome: A Pilot Study
}

\author{
Alessandra Meloni, ${ }^{1}$ Francesco Palmas, ${ }^{2}$ Luigi Barberini ${ }^{D},{ }^{3}$ Rossella Mereu, ${ }^{1}$ \\ Sara Francesca Deiana, ${ }^{1}$ Maria Francesca Fais, ${ }^{1}$ Antonio Noto $\left(\mathbb{D},{ }^{4}\right.$ Claudia Fattuoni $\mathbb{D}^{2}{ }^{2}$ \\ Michele Mussap, ${ }^{5}$ Antonio Ragusa, ${ }^{6}$ Angelica Dessì ${ }^{(D},{ }^{4}$ Roberta Pintus, ${ }^{4}$ Vassilios Fanos, ${ }^{4}$ \\ and Gian Benedetto Melis ${ }^{1}$
}

\author{
${ }^{1}$ Department of Surgical Sciences, Division of Gynaecology and Obstetrics, University of Cagliari, Cagliari, Italy \\ ${ }^{2}$ Department of Chemical and Geological Sciences, University of Cagliari, Cagliari, Italy \\ ${ }^{3}$ Department of Medical Sciences and Public Health, University of Cagliari, Cagliari, Italy \\ ${ }^{4}$ Department of Surgical Sciences, Neonatal Intensive Care Unit, Puericulture Institute and Neonatal Section, \\ Azienda Ospedaliera Universitaria, Cagliari, Italy \\ ${ }^{5}$ Laboratory Medicine Service, IRCCS AOU San Martino-IST, University-Hospital, Genoa, Italy \\ ${ }^{6}$ Maternal Neonatal Department, Division of Gynaecology and Obstetrics, Massa Carrara Hospital, Carrara, Italy
}

Correspondence should be addressed to Claudia Fattuoni; cfattuon@unica.it

Received 17 October 2017; Accepted 24 December 2017; Published 4 February 2018

Academic Editor: Vincent Sapin

Copyright ( 2018 Alessandra Meloni et al. This is an open access article distributed under the Creative Commons Attribution License, which permits unrestricted use, distribution, and reproduction in any medium, provided the original work is properly cited.

\begin{abstract}
Since pathologies and complications occurring during pregnancy and/or during labour may cause adverse outcomes for both newborns and mothers, there is a growing interest in metabolomic applications on pregnancy investigation. In fact, metabolomics has proved to be an efficient strategy for the description of several perinatal conditions. In particular, this study focuses on premature rupture of membranes (PROM) in pregnancy at term. For this project, urine samples were collected at three different clinical conditions: out of labour before PROM occurrence (Ph1), out of labour with PROM (Ph2), and during labour with PROM (Ph3). GC-MS analysis, followed by univariate and multivariate statistical analysis, was able to discriminate among the different classes, highlighting the metabolites most involved in the discrimination.
\end{abstract}

\section{Introduction}

The early diagnosis of pregnancy-related complications and the prediction of pregnancy outcome are considered strategic clinical goals to ensure the health of mothers and of their babies. Among these, premature rupture of membranes (PROM) consists of the rupture of the foetal membranes before the onset of labour. It can be observed at any gestational age [1] and occurs in approximately $10 \%$ of pregnant women and in roughly $40 \%$ of preterm deliveries [2]. Foetal membranes are of pivotal importance because they offer a robust barrier against infection ascending from the reproductive tract; after their rupture, both the mother and foetus are at risk of infection and other complications. The most commonly diagnosed maternal infections in case of PROM are chorioamnionitis and endometritis, which may be further promoted by frequent vaginal exams and the presence of meconium in the amniotic fluid (AF) [3]. Foetal complications of PROM include neonatal sepsis, abnormal foetal presentation, cord prolapse or compression, and abruptio placentae, and these may increase the risk of neonatal intraventricular haemorrhage, leading to neurodevelopmental disability as a direct consequence [4]. Therefore, from PROM, diagnosis may derive different medical procedures such as hospitalization, antibiotic prophylaxis, and induction of labour through oxytocin $[5,6]$, that is, increased medicalization and caesarean section rates. These practices may in turn affect several women in the positive experience of birth [7]. Moreover, given the increasing antibiotic resistance, alerts have been issued about the use and abuse of prophylactic antibiotic administration [8]. Indeed, growing evidences on this phenomenon suggest possible short- and long-term 
risks on maternal and foetal microbiota, resulting in longterm sequelae such as obesity, food allergies and intolerances, autoimmune diseases, and possible neurodevelopmental involvement. Although the exact aetiology is unclear, known factors are collagen remodelling, apoptosis [9], increased transcription of matrix metalloproteinases (MMP) such as MMP9, AF apoptotic activators $[10,11]$, and polymorphism promoter of tumour necrosis factor $\alpha$ (TNF- $\alpha$ ), interleukin-1 (IL-1), and MMP1 [12, 13]. Notably, there is no universally accepted method for the diagnosis of PROM. Strategies currently performed consist of sterile speculum examination and nitrazine or fern tests [14], while ultrasound is useful to identify an AF reduction in the case of suspect membrane rupture [15]. Albeit these techniques have been employed for more than 60 years, the nitrazine test has been recently discouraged [16]. Moreover, since the AF concentration of several biomarkers is higher than that in normal vaginal secretion, many studies investigated the diagnostic value of vaginal AF for an early and accurate diagnosis of PROM. As a result, a number of potential biomarkers including prolactin, $\alpha$-fetoprotein (AFP), $\beta$-subunit of human chorionic gonadotropin $(\beta$-HCG), foetal fibronectin, diamine oxidase, lactate, creatinine, urea, and insulin growth factor-binding protein-1 (IGF-BP1) have been proposed and tested [17, 18]. In particular, IGF-BP1 is the major protein in AF, and its presence confirms AF contamination in vaginal secretions. Interestingly, most of these biomarkers seem to accurately distinguish patients with intact membranes from those with unequivocal membrane rupture; however, they are not routinely applied due to their complex procedure, cost, and low sensitivities in patients with equivocal rupture. For these reasons, further investigations are necessary for the development of novel, versatile, and timely accurate diagnostic means. Among the most recent methods of investigation, metabolomics was successfully applied to describe the different molecular profiles arising over gestation [19] as well as the dynamics responsible for maternal and foetal unfavourable outcomes and labour and delivery complications $[20,21]$. As a matter of fact, characterisation of the metabolic profile in various biological fluids such as AF, urine, maternal and cord blood, and vaginal secretions is considered one of the most promising and attractive tools for an early and accurate identification of several maternal morbidities and childbirth events [22]. Therefore, the present work applied a metabolomic approach to investigate the urinary metabolome in relation to PROM occurrence and labour. To pursue this goal, metabolic differences were observed in women with intact membranes and out of labour, with PROM and prior to labour, and with PROM and during labour.

\section{Materials and Methods}

2.1. Study Design and Population. Between October 2013 and July 2014, thirty-eight pregnant women at term, age 29-42 years (gestational age (GA) between 38 weeks +0 day and 40 weeks +4 days) with single, low-risk pregnancy, and foetuses in vertex presentation were admitted at the Unit of Obstetrics and Gynaecology of the University-Hospital of
Cagliari and enrolled in this study. Patients gave written informed consent at the time of admission. The study was conducted in accordance with the Declaration of Helsinki (1964) and previously approved by the local ethics committee. Women were divided into 3 phenotypical groups. The first phenotypical group Ph1 consisted of 11 healthy pregnant women enrolled long time before labour (out of labour and intact membranes), these women were successively admitted with PROM. Group Ph2 consisted of 10 pregnant women with PROM (out of labour and PROM). Group Ph3 consisted of 17 pregnant women with prior diagnosis of PROM and in labour (in labour and PROM). Diagnosis of PROM was based on women's history, direct visualization of fluid leakage, and speculum examination. When direct visualization of AF loss was unreliable, a qualitative immunochromatographic dipstick test for AF IGF-BP1 presence, together with ultrasound AF evaluation, was performed. Diagnosis was then retrospectively confirmed after delivery.

2.2. Sample Collection and Storage. A spot urine sample was collected from each pregnant woman enrolled in the study; hence, a total of 38 samples were collected and analysed. For the Ph1 group, the urine was collected 3-14 days before delivery (median value 7 days, interquartile range (IQR) 511 days); for group Ph2, 15-50 hours before delivery (median value 28.5 hours, IQR 25.5-40 hours); and for group Ph3, 0.25-19 hours before delivery (median value 10 hours, IQR 5-13 hours). Sampling was performed through a sterile, preservative-free urine beaker equipped with a transfer device (VACUETTE ${ }^{\circledR}$, Greiner Bio-One International $\mathrm{GmbH}$, Kremsmünster, Austria) which allowed for the automatic filling of a vacuum urine tube without any external contamination. In detail, urine passes from the beaker to the vacuum tube by pushing it into the transfer device. The tube is then automatically filled for about $10 \mathrm{~mL}$. After collection, all tubes were centrifuged and the supernatant was immediately frozen and stored at $-80^{\circ} \mathrm{C}$ until analysis.

2.3. Sample Preparation. Urine samples were treated as previously described [23]. In brief, specimens were thawed at room temperature, $150 \mu \mathrm{L}$ was transferred into Eppendorf tubes, and $800 \mu \mathrm{L}$ of urease solution $(1 \mathrm{mg} / \mathrm{mL})$ was added. Following 30-minute sonication and deproteinisation, samples were centrifuged at $14000 \mathrm{rpm}$ and $1200 \mu \mathrm{L}$ of the supernatant was dried in a vacuum centrifuge overnight. $30 \mu \mathrm{L}$ of a $0.24 \mathrm{M}$ solution of methoxylamine hydrochloride in pyridine was added to each vial and kept at room temperature for 17 hours. $30 \mu \mathrm{L}$ of $N$-methyl- $N$-trimethylsilyltrifluoroacetamide (MSTFA) was added and kept at room temperature for 1 hour. The derivatized samples were diluted with $600 \mu \mathrm{L}$ of a tetracosane solution in hexane $(0.01 \mathrm{mg} / \mathrm{mL})$ just before GC-MS analysis.

2.4. Sample Analysis and Data Processing. The derivatized samples were analysed by using a global unbiased mass spectrometry-based platform with GC-MS incorporating an Agilent 5975C interfaced to a GC 7820 (Agilent Technologies, Palo Alto, CA, USA). The system was equipped with a DB-5 ms column (Agilent J\&W Scientific, Folsom, CA, 
USA); injection temperature was set at $230^{\circ} \mathrm{C}$ and detector temperature at $280^{\circ} \mathrm{C}$. Carrier gas (helium) flow rate was equal to $1 \mathrm{~mL} / \mathrm{min}$. GC oven starting temperature programme was $90^{\circ} \mathrm{C}$ with $1 \mathrm{~min}$ hold time and ramping at a rate of $10^{\circ} \mathrm{C}$ per minute, reaching a final temperature of $270^{\circ} \mathrm{C}$ with $7 \mathrm{~min}$ hold time. $1 \mu \mathrm{L}$ of the derivatized sample was injected in split $(1: 20)$ mode. After a solvent delay of $3 \mathrm{~min}$, mass spectra were acquired in full-scan mode using 2.28 scans per second, with a mass range of 50-700 Amu. Each acquired chromatogram was analysed by means of the free software Automated Mass Spectral Deconvolution and Identification System (AMDIS) (http://chemdata.nist.gov/ mass-spc/amdis). Each peak was identified by comparing the corresponding mass spectra and retention times with those stored in an in-house library including 255 metabolites. Other metabolites were identified by using the National Institute of Standards and Technology's mass spectral database (NIST08) and Golm Metabolome Database (GMD; http://gmd.mpimp-golm.mpg.de/). Metabolites were considered positively identified with a match factor $\geq 70 \%$. For lower values, metabolites were labelled as "unknown." This analysis produced a matrix spreadsheet containing 84 metabolites, 77 identified and 7 unknown, to be submitted to chemometric analysis.

2.5. Statistical Analysis. Sample size was adequate to assure the minimum precision requested for a pilot study [24]. All analyses were performed on MetaboAnalyst 3.0 (http:// www.metaboanalyst.ca/) [25]. Both univariate and multivariate approaches were applied. In particular, univariate analysis consisted in analysis of variance (ANOVA) with Tukey's honestly significant difference (HSD) post hoc test with a false discovery rate (FDR) cut-off of 0.05 for analysis on more than two phenotypes, while Student's $t$-test with $p<0.05$ cutoff was conducted for 2-phenotype models. Multivariate analysis was performed by means of the supervised partial least squares discriminant analysis (PLS-DA) in order to identify important variables with discriminative power, named variable importance in projection (VIP), and their trends. PLS-DA models were then submitted to a 10 -fold cross-validation (CV) method for the evaluation of statistical parameters (accuracy, $R^{2}$, and $Q^{2}$ ) and the determination of the number of components that best describe the models. The PLS-DA model was further validated by permutation tests based on prediction accuracy $(n=100$ and $p<0.01)$. The power analysis test was performed to determine the sample size required to detect a statistically significant difference between the two populations with a given degree of confidence $(\mathrm{FDR}=0.1)$.

\section{Results and Discussion}

3.1. Three-Phenotype Model. First, statistical analysis and comparisons were conducted on all 3 different phenotypes at the same time. Applying ANOVA with Tukey's HSD post hoc test on the three classes $\mathrm{Ph} 1, \mathrm{Ph} 2$, and $\mathrm{Ph} 3$ resulted in 58 significant metabolites. For discussion purpose, these compounds were divided into 5 groups corresponding to different chemical classes: carbohydrates (Carb), oxidised carbohydrates (Ox), amino acids (AA), sugar related (SR), and miscellaneous (Misc). Metabolites' list for such calculations is reported in Table 1.

PLS-DA of the same classes produced an unsatisfactory model scoring accuracy $=0.67, R^{2}=0.88$, and $Q^{2}=0.33$. Moreover, the permutation test delivered a $p=0.04$ (Figure 1)

3.2. PROM Model: Ph1 (Out of Labour, Intact Membranes) versus Ph2 (Out of Labour, PROM). In order to investigate the metabolic differences caused solely by the rupture of membranes, the $t$-test was performed on Ph1 and Ph2 groups. This calculation highlighted 9 significant metabolites: galactose, uric acid, 3,4-dihydroxybutyric acid, galactitol, alanine, lysine, 4-hydroxyphenylacetic acid, serine, and hydroxyproline dipeptide. Notably, the entire set of metabolites resulted more abundant in Ph1, except for uric acid. This means that these metabolites are significantly consumed during PROM events. Also in this case, PLS-DA produced an unsuitable model with accuracy $=0.76, R^{2}=0.43, Q^{2}=0.29$, and $p=0.09$. However, the first 9 metabolites from PLS-DA correspond to those obtained from the $t$-test, and higher levels for all metabolites were observed in the Ph1 group. Power analysis calculated the number of samples for a predictive power of 0.83 as 120 per group (Figure 2).

3.3. Labour Model: Ph2 (Out of Labour, PROM) versus Ph3 (in Labour, PROM). Labour effects on the metabolome were highlighted by the comparison of $\mathrm{Ph} 2$ and $\mathrm{Ph} 3$ groups. The $t$ test indicated 60 significant metabolites between the two groups of interest (Table 2).

Unsatisfactory PLS-DA results delivered a model with accuracy $=0.88, R^{2}=0.96, Q^{2}=0.54$, and $p=0.04$. Scores and VIP plots are shown in Figure 3.

The majority of the metabolites responsible for such phenotype discrimination showed higher levels in the $\mathrm{Ph} 3$ group (in labour with PROM), while phosphate, lactose, and uric acid were more abundant in Ph2. Therefore, the metabolites are mainly produced during labour. Power analysis indicated better results for this model: a predictive power of 0.83 for 50 samples per group (Figure 4).

\section{Discussion}

Currently, assessment of PROM is mainly based on external genital leakage and/or direct observation of AF loss by direct visualization through speculum examination. Other options are biochemical tests and ultrasound AF evaluation, but none of these strategies may securely confirm diagnosis [26]; subsequently, confirmation of PROM often occurs during labour. Nevertheless, it is of crucial importance to make accurate and timely diagnosis in order to define appropriate clinical interventions, hence to avoid complications for the patients. In this study, although multivariate analysis could not reach statistical significance, univariate calculations identified several discriminant metabolites for the 3 phenotypes; 35 out of 58 could be distinguished between at least two comparisons. Therefore, the metabolic profiles are indeed altered due to PROM and/or labour. Nevertheless, more samples are necessary to provide a holistic model that may describe such 
TABLE 1: Statistically significant $(F D R<0.05)$ metabolites from univariate analysis (ANOVA with Tukey's HSD post hoc test) of the three classes Ph1, Ph2, and Ph3.

\begin{tabular}{|c|c|c|c|c|}
\hline Metabolite & Chemical class & $p$ value & FDR & Tukey's HSD \\
\hline cis-Aconitic acid & Misc & $2.01 E-03$ & 0.00015894 & $2-1,3-1,3-2$ \\
\hline 2,3,4,5-Tetrahydroxypentanoic acid 1,4-lactone & Ox & $8.63 E-02$ & 0.00026357 & $2-1,3-1,3-2$ \\
\hline Erythronic acid & Ox & $1.20 E-01$ & 0.00026357 & $2-1,3-1,3-2$ \\
\hline 3-Hydroxybutyric acid & Misc & $1.75 E-01$ & 0.00026357 & $3-1,3-2$ \\
\hline 3,4-Dihydroxybutyric acid & Misc & $1.95 E-01$ & 0.00026357 & $2-1,3-2$ \\
\hline Glucaric acid & Ox & $2.00 E-01$ & 0.00026357 & $2-1,3-2$ \\
\hline Unknown C & Misc & $2.91 E-01$ & 0.00029809 & $3-1,3-2$ \\
\hline Pseudouridine & Misc & $3.02 \mathrm{E}-01$ & 0.00029809 & $3-1,3-2$ \\
\hline Erythritol & SR & $3.98 E-01$ & 0.00030706 & $3-1,3-2$ \\
\hline Gulonic acid & Ox & $4.00 E-01$ & 0.00030706 & $3-1,3-2$ \\
\hline Monosaccharide 1886 & SR & $4.28 E-01$ & 0.00030706 & $3-1,3-2$ \\
\hline Pyroglutamic acid & Misc & $5.02 E-01$ & 0.00033075 & $2-1,3-2$ \\
\hline Arabitol & SR & $5.44 E-01$ & 0.00033075 & $3-1,3-2$ \\
\hline Fucose & Carb & $5.94 E-01$ & 0.00033536 & $3-1,3-2$ \\
\hline Ribitol & SR & $6.53 E-01$ & 0.00034397 & $2-1,3-2$ \\
\hline Tyrosine & AA & 0.00011408 & 0.00056327 & $2-1,3-2$ \\
\hline Xylobiose & Carb & 0.00016349 & 0.00075975 & $3-2$ \\
\hline 3,4,5-Trihydroxypentanoic acid & Misc & 0.00023492 & 0.0010311 & $2-1,3-2$ \\
\hline Histidine & AA & 0.00027084 & 0.0011261 & $2-1,3-2$ \\
\hline Gluconic acid & Ox & 0.00040226 & 0.0015889 & $3-2$ \\
\hline Serine & $\mathrm{AA}$ & 0.00050574 & 0.0019025 & $2-1,3-2$ \\
\hline Lysine & $\mathrm{AA}$ & 0.0005569 & 0.0019615 & $2-1,3-2$ \\
\hline Monosaccharide E & SR & 0.00058834 & 0.0019615 & $3-2$ \\
\hline Xylitol & SR & 0.00059589 & 0.0019615 & $3-1,3-2$ \\
\hline Phenylalanine & $\mathrm{AA}$ & 0.00089953 & 0.0028425 & $2-1,3-2$ \\
\hline Threonine & AA & 0.00098451 & 0.0029914 & $2-1,3-2$ \\
\hline Quinolinic acid & Misc & 0.0012588 & 0.003683 & $2-1,3-2$ \\
\hline 4-Deoxythreonic acid & SR & 0.0014511 & 0.0040942 & $3-2$ \\
\hline Cystine & $\mathrm{AA}$ & 0.0015954 & 0.004346 & $3-2$ \\
\hline Succinic acid & Misc & 0.0024091 & 0.0063441 & $3-2$ \\
\hline Alanine & $\mathrm{AA}$ & 0.0027785 & 0.0070807 & $2-1,3-2$ \\
\hline$N$-Acetylglucosamine & SR & 0.0035822 & 0.0088435 & $3-2$ \\
\hline Phosphate & Misc & 0.0037469 & 0.0089697 & $3-2$ \\
\hline Ribonic acid & $\mathrm{Ox}$ & 0.0042205 & 0.0098065 & $3-2$ \\
\hline 2-Amino-6-hydroxy-7-methyl-7H-purine & Misc & 0.0043539 & 0.0098274 & $3-1,3-2$ \\
\hline Hydroxyproline dipeptide & AA & 0.0048016 & 0.010537 & $2-1,3-2$ \\
\hline Maltose & Carb & 0.0050599 & 0.010804 & $2-1,3-1$ \\
\hline Glutamine & AA & 0.0055744 & 0.011425 & $2-1,3-2$ \\
\hline Glycine, $N$-4-hydroxybenzoyl & Misc & 0.0057479 & 0.011425 & $2-1,3-2$ \\
\hline Threonic acid & $\mathrm{Ox}$ & 0.005785 & 0.011425 & $2-1,3-2$ \\
\hline 2-O-Glycerol-galactopyranoside & SR & 0.006708 & 0.012925 & $2-1,3-2$ \\
\hline Lactose & Carb & 0.0077141 & 0.01451 & $2-1,3-2$ \\
\hline Ribose & Carb & 0.0088787 & 0.016312 & $3-2$ \\
\hline Citric acid & Misc & 0.0092274 & 0.016567 & $3-2$ \\
\hline Galactose & Carb & 0.010113 & 0.017412 & $3-1$ \\
\hline 4-Hydroxyphenylacetic acid & Misc & 0.010139 & 0.017412 & $2-1,3-2$ \\
\hline
\end{tabular}


TABle 1: Continued.

\begin{tabular}{lcccc}
\hline Metabolite & Chemical class & $p$ value & FDR & Tukey's HSD \\
\hline Glucose & Carb & 0.012002 & 0.020173 & $2-1$ \\
Glycine, $N$-4-hydroxybenzoyl derivative & Misc & 0.018028 & 0.029671 & $3-2$ \\
Uric acid & Misc & 0.0197 & 0.031761 & 0.032514 \\
Threitol & SR & 0.020579 & 0.033314 & $3-2$ \\
Galactitol & SR & 0.021507 & 0.035603 & $2-1$ \\
Hippuric acid & Misc & 0.023726 & 0.035603 & $3-2$ \\
Creatinine & Misc & 0.023885 & 0.038588 & $3-1,3-2$ \\
2,4-Dihydroxybutyric acid & Misc & 0.026377 & 0.039336 & $3-2$ \\
2-Ketogluconic acid & Ox & 0.027386 & 0.039753 & $3-1$ \\
Arabinose & Carb & 0.028179 & 0.040153 & $3-2$ \\
Sedoheptulose & Carb & 0.028971 & 0.046565 \\
4-Deoxyerythronic acid & Ox & 0.034187 & $3-2$ \\
\hline
\end{tabular}

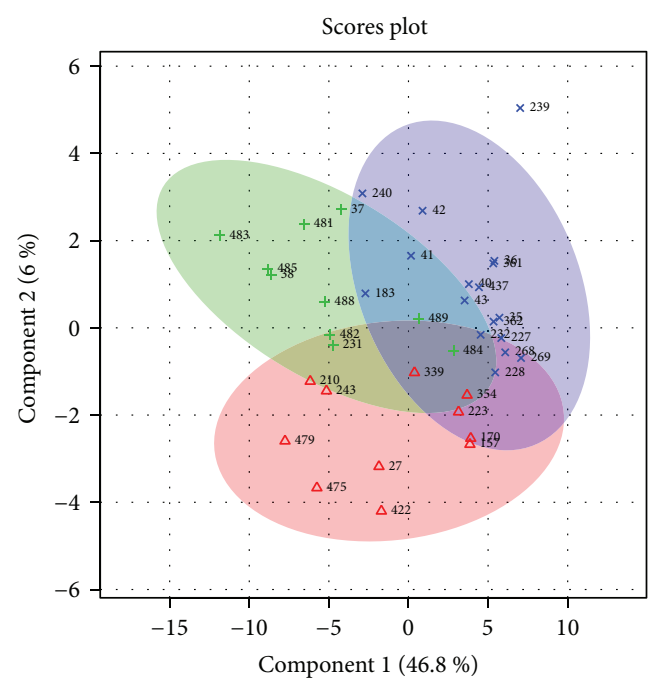

$\triangle 1$

$+2$

$\times 3$

(a)

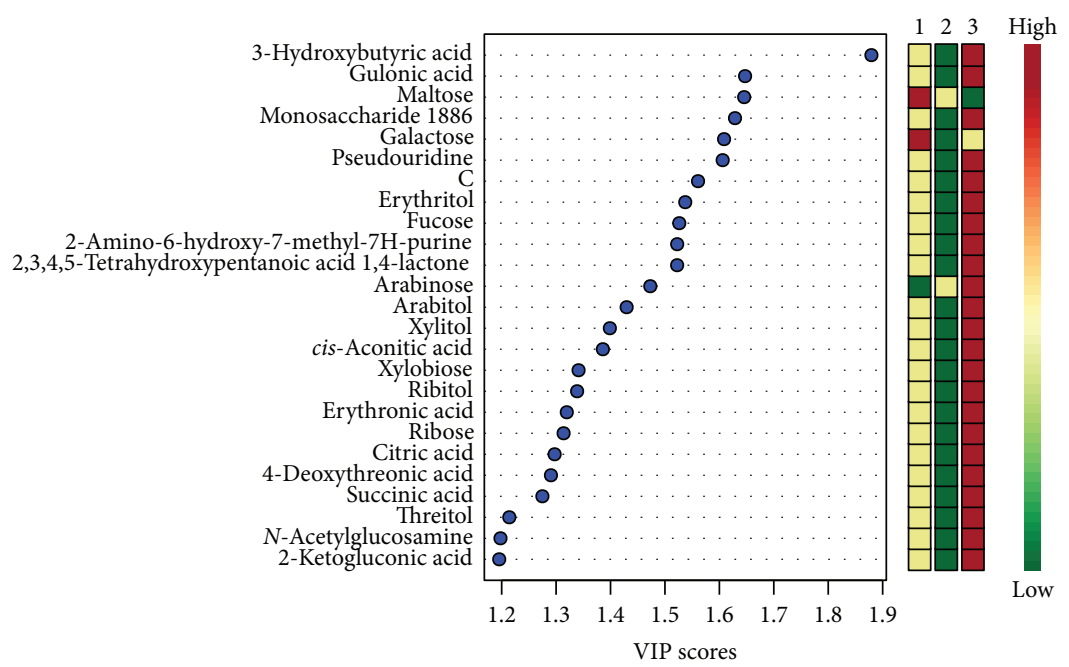

(b)

Figure 1: (a) 2D score plot showing PLS-DA discrimination between Ph1 (red, out of labour, intact membranes), Ph2 (green, out of labour, PROM), and Ph3 (blue, in labour, PROM) and (b) the corresponding VIP score plot.

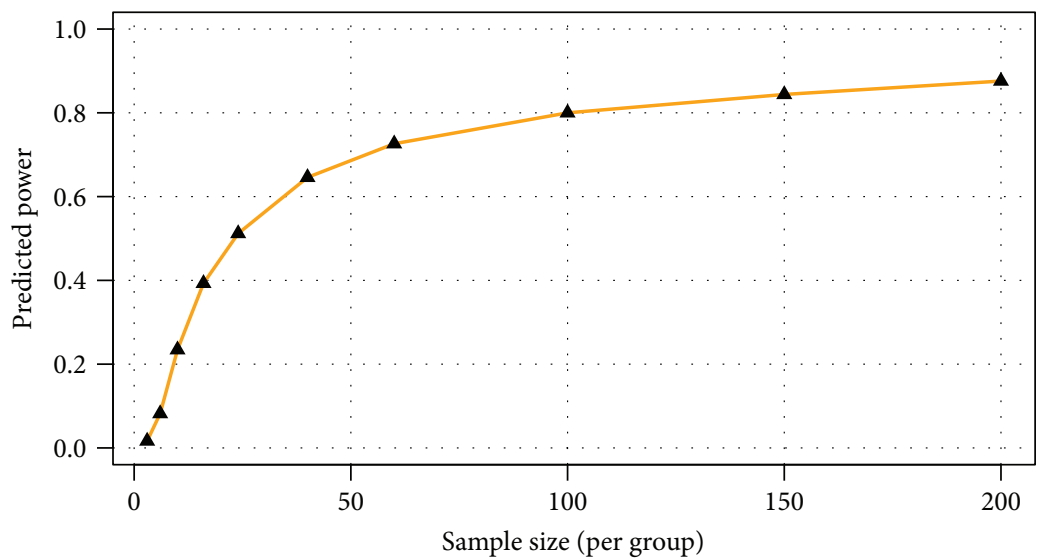

FIgURE 2: Power analysis calculations indicated a predictive power of 0.83 for 120 samples per group and an FDR of 0.1 . 
TABLE 2: Statistically significant $(\mathrm{FDR}<0.05)$ metabolites from the $t$-test of the classes $\mathrm{Ph} 2$ versus $\mathrm{Ph} 3$.

\begin{tabular}{|c|c|c|c|c|}
\hline Metabolite & Chemical class & $p$ value & FDR & $\mathrm{Ph} 2$ versus $\mathrm{Ph} 3$ \\
\hline Gulonic acid & $\mathrm{Ox}$ & $7.31 E-04$ & $5.63 E-01$ & Down \\
\hline Erythronic acid & Ox & $3.00 E-02$ & $7.03 E-01$ & Down \\
\hline 2,3,4,5-Tetrahydroxypentanoic acid 1.4-lactone & Ox & $3,53 \mathrm{E}-03$ & $7.03 E-01$ & Down \\
\hline cis-Aconitic acid & Misc & $4,44 \mathrm{E}-02$ & $7.03 E-01$ & Down \\
\hline Glucaric acid & Ox & $4.76 E-03$ & $7.03 E-01$ & Down \\
\hline Pyroglutamic acid & Misc & $5.48 E-02$ & $7.03 E-01$ & Down \\
\hline Ribitol & SR & $9.12 E-02$ & $9.12 E-01$ & Down \\
\hline Arabitol & SR & $9.47 E-02$ & $9.12 E-01$ & Down \\
\hline Fucose & Carb & $1.07 E-01$ & $9.14 E-02$ & Down \\
\hline Unknown C & Misc & $1.34 E-01$ & 0.00010306 & Down \\
\hline Tyrosine & AA & $1.52 E-01$ & 0.0001065 & Down \\
\hline Erythritol & SR & $1.87 E-01$ & 0.00011968 & Down \\
\hline Pseudouridine & Misc & $2.55 E-01$ & 0.00015105 & Down \\
\hline 3,4-Dihydroxybutyric acid & Misc & $4.19 E-01$ & 0.00023071 & Down \\
\hline Monosaccharide 1886 & Carb & $5.13 E-01$ & 0.00026311 & Down \\
\hline Xylobiose & Carb & $5.80 E-01$ & 0.00027903 & Down \\
\hline Gluconic acid & Ox & $6.60 E-01$ & 0.00029457 & Down \\
\hline Histidine & AA & $7.26 E-01$ & 0.00029457 & Down \\
\hline 4-Deoxythreonic acid & Ox & $7.27 E-01$ & 0.00029457 & Down \\
\hline 3-Hydroxybutyric acid & Misc & $9.00 E-01$ & 0.00034512 & Down \\
\hline Phenylalanine & AA & 0.00010095 & 0.00034512 & Down \\
\hline Monosaccharide E & Carb & 0.0001043 & 0.00034512 & Down \\
\hline 3,4,5-Trihydroxypentanoic acid & Ox & 0.00010624 & 0.00034512 & Down \\
\hline Sedoheptulose & Carb & 0.00010757 & 0.00034512 & Down \\
\hline Quinolinic acid & Misc & 0.00012733 & 0.00039216 & Down \\
\hline Xylitol & SR & 0.00017953 & 0.00053168 & Down \\
\hline Phosphate & Misc & 0.00020953 & 0.00059755 & Up \\
\hline Lysine & AA & 0.000373 & 0.0010074 & Down \\
\hline Succinic acid & Misc & 0.00037941 & 0.0010074 & Down \\
\hline Ribose & Carb & 0.00049401 & 0.001268 & Down \\
\hline Threonine & AA & 0.00055591 & 0.0013069 & Down \\
\hline Cystine & AA & 0.00056022 & 0.0013069 & Down \\
\hline 2-Amino-6-hydroxy-7-methyl-7H-purine & Misc & 0.00056835 & 0.0013069 & Down \\
\hline Serine & AA & 0.00057708 & 0.0013069 & Down \\
\hline$N$-Acetylglucosamine & SR & 0.00074989 & 0.0016498 & Down \\
\hline Citric acid & Misc & 0.0009237 & 0.0019757 & Down \\
\hline Ribonic acid & Ox & 0.0013077 & 0.0027213 & Down \\
\hline Glutamine & AA & 0.0014072 & 0.0028515 & Down \\
\hline Lactose & Carb & 0.0020445 & 0.0040365 & Up \\
\hline Glucose & Carb & 0.0024816 & 0.004777 & Down \\
\hline Hydroxyproline dipeptide & AA & 0.0028275 & 0.0053102 & Down \\
\hline Glycine, $N$-4-hydroxybenzoyl & Misc & 0.0038577 & 0.0070724 & Down \\
\hline 2-Ketogluconic acid & Ox & 0.0045002 & 0.0080016 & Down \\
\hline 2,4-Dihydroxybutyric acid & Misc & 0.0045723 & 0.0080016 & Down \\
\hline Threonic acid & Ox & 0.0047704 & 0.0081627 & Down \\
\hline 4-Hydroxyphenylacetic acid & Misc & 0.0051136 & 0.0085598 & Down \\
\hline
\end{tabular}


TABLE 2: Continued.

\begin{tabular}{lcccc}
\hline Metabolite & Chemical class & $p$ value & FDR & Ph2 versus Ph3 \\
\hline Alanine & AA & 0.0052474 & 0.0085968 & Down \\
2-O-Glycerol-galactopyranoside & SR & 0.0071983 & 0.011547 & Down \\
Threitol & SR & 0.0091347 & 0.014354 & Down \\
Glycine, $N$-4-hydroxybenzoyl derivative & Misc & 0.010305 & 0.015869 & 0.019557 \\
Creatinine & Misc & 0.012953 & 0.020799 & Down \\
Uric acid & Misc & 0.014046 & 0.02117 & Up \\
3-Methylhistidine & AA & 0.014579 & 0.02117 & Down \\
Glyceromannoheptonic acid & Ox & 0.014847 & 0.022086 & Down \\
Hippuric acid & Misc & 0.015776 & 0.022232 & Down \\
Inositol & SR & 0.016169 & 0.023086 & Down \\
Arabinose & Carb & 0.017089 & 0.023587 & Down \\
4-Deoxyerythronic acid & Ox & 0.017767 & 0.031591 & Down \\
Ethanolamine & Misc & 0.024206 & 0.033697 & Down \\
Mannitol & SR & 0.026258 & &
\end{tabular}

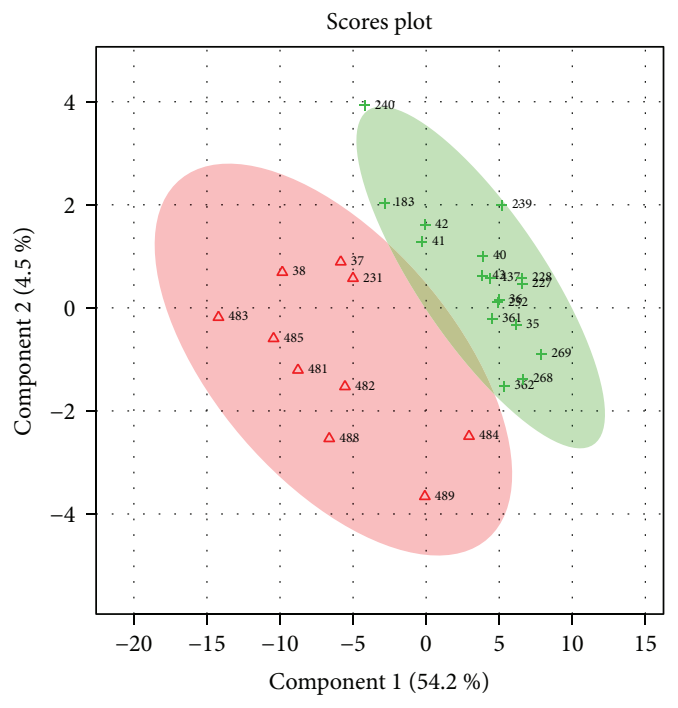

$$
\triangle 2
$$

(a)

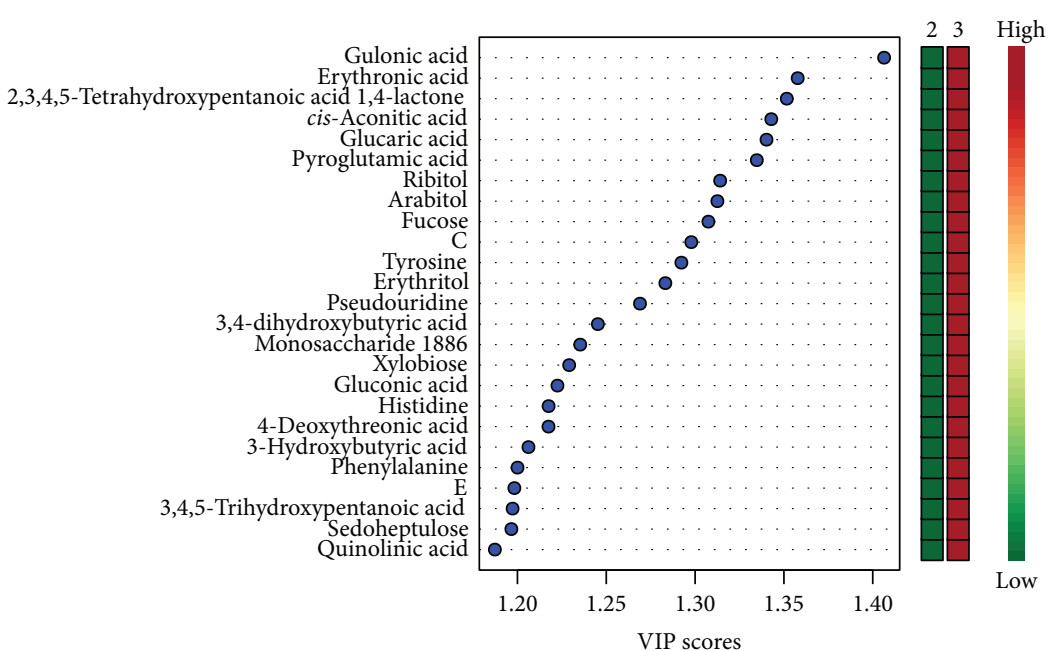

(b)

Figure 3: (a) Score plot showing the separation and clustering between Ph2 (2, triangles) and Ph3 (3, crosses) and (b) the corresponding VIP score plot.

heterogeneous and particular conditions. In particular, the separation between $\mathrm{Ph} 2$ and $\mathrm{Ph} 3$, which is characterised by PROM and differs on the onset of labour, seems to require the lowest number of samples and showed the highest number of discriminant metabolites through the $t$-test. For these reasons, it may be hypothesised that labour event affects more the system than PROM.

Furthermore, the fact that the PROM model (Ph1 versus $\mathrm{Ph} 2$ ) produced the lowest values for the statistical parameters may also suggest the eventuality that these two phenotypes are actually similar. Indeed, PROM per se may not represent a pathological event in the absence of complications. Therefore, further analysis should consider the outcome of delivery in PROM subjects. In detail, by analysing the alteration due to PROM, the almost totality of the discriminant compounds showed higher levels in the intact membrane group; hence, the discriminant compounds are significantly consumed in the case of broken membranes. Unfortunately, discriminant metabolites are of difficult interpretation. By observing the labour model (Ph2 versus Ph3), in labour subjects excreted higher amounts of the majority of the significant metabolites, while phosphate, lactose, and uric acid are excreted in lower amounts. Among the several significant metabolites, 3,4dihydroxybutyric acid is a product of the oxidative metabolism of fatty acids and its increase can be observed in case of inflammations to satisfy the need for a surplus of energy 


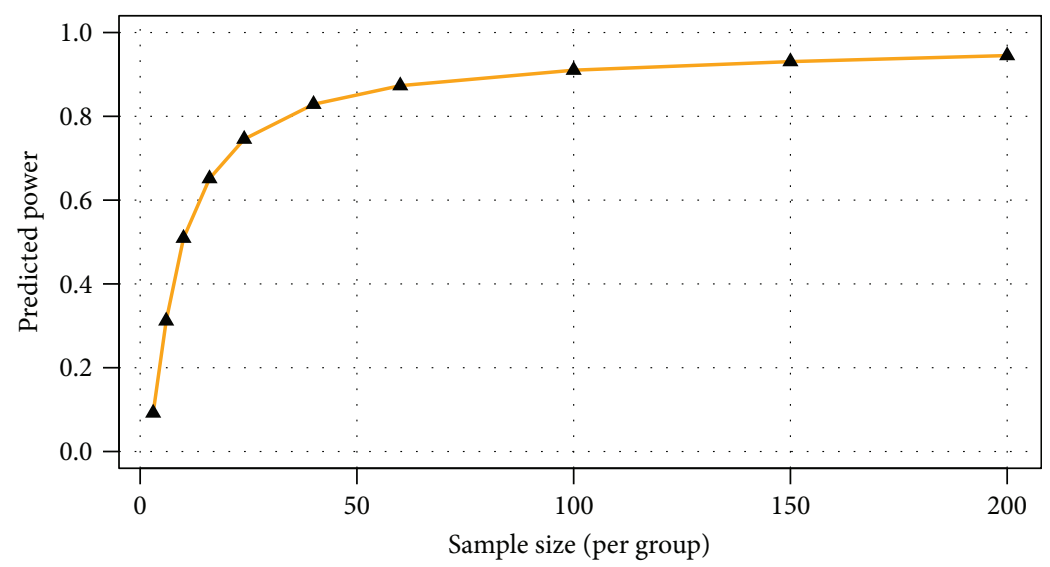

FIGURE 4: Power analysis calculations indicated a predictive power of 0.83 for 50 samples per group and an FDR of 0.1 .

due to stress conditions. Interestingly, it seems that inflammation processes may trigger preterm delivery [27]. Another sign of oxidative stress is the higher levels of glucuronic, gulonic, glucaric, and gluconic acids and other oxidised carbohydrates (Table 2) which derive from oxidative conversion [27, 28]. Moreover, the discriminant uric acid is involved in antioxidant activity in blood toward peroxyl radicals, which are released due to oxidative stress and ROS overproduction in labour [29]. By comparing the results from labour model calculations to those reported [30], only cis-aconitic acid showed an analogous trend. Indeed, this metabolite showed an upregulation characterising the active labour phase. cis-Aconitic acid is a wellknown intermediate of the tricarboxylic acid (TCA) cycle, and its high level may be explained by the increased energy demand during labour.

\section{Conclusions}

GC-MS-based analysis of pregnant women's urinary metabolome could deliver interesting information about PROM and labour events. Most pregnant women at term will start labour in the next 24 hours after PROM; in fact, clinical guidelines recommend to wait at least 24 hours before labour induction. Intriguingly, further studies may investigate metabolic profiles associated with the timely spontaneous onset of labour to appropriately select women for labour induction.

Although the number of samples was not optimal for solid evidence, univariate chemometric analysis was able to discriminate among the different conditions, highlighting variation in the phenotypes' metabolome. An interesting feature of these data is that labour conditions seem to have greater influence on the system than the actual PROM occurrence, suggesting that further studies are in need for this delicate diagnosis. Nevertheless, a metabolomic approach on appropriate sample size may be a promising tool for research in the field of obstetrics.

\section{Conflicts of Interest}

The authors have no conflict of interest to declare.

\section{Authors' Contributions}

Alessandra Meloni and Francesco Palmas contributed equally to this work.

\section{Acknowledgments}

This work was supported by Legge Regionale 7 (July 08, 2007) (CRP60413/2012): "Promotion of scientific research and technologic innovation in Sardinia" and "Metabolomic study for new predictive and prognostic biomarkers in the diagnosis of labour." Francesco Palmas gratefully acknowledges the Sardinia Regional Government for the financial support of his $\mathrm{PhD}$ scholarship (P.O.R. Sardegna F.S.E. Operational Programme of the Autonomous Region of Sardinia, European Social Fund 2007-2013-Axis IV Human Resources, Objective 1.3, Line of Activity 1.3.1.).

\section{References}

[1] S. Parry and J. F. Strauss, "Premature rupture of the fetal membranes," The New England Journal of Medicine, vol. 338, no. 10, pp. 663-670, 1998.

[2] D. Kumar, R. M. Moore, B. M. Mercer, J. M. Mansour, R. W. Redline, and J. J. Moore, "The physiology of fetal membrane weakening and rupture: insights gained from the determination of physical properties revisited," Placenta, vol. 42, pp. 59-73, 2016.

[3] A. B. Caughey, J. N. Robinson, and E. R. Norwitz, "Contemporary diagnosis and management of preterm premature rupture of membranes," Reviews in Obstetrics \& Gynecology, vol. 1, no. 1, pp. 11-22, 2008.

[4] T. M. Medina and D. A. Hill, "Preterm premature rupture of membranes: diagnosis and management," American Family Physician, vol. 73, no. 4, pp. 659-664, 2006.

[5] L. Selin, E. Almström, G. Wallin, and M. Berg, "Use and abuse of oxytocin for augmentation of labor," Acta Obstetricia et Gynecologica Scandinavica, vol. 88, no. 12, pp. 1352-1357, 2009.

[6] K. S. J. Oláh and P. J. Steer, "The use and abuse of oxytocin," The Obstetriciane Gynaecologist, vol. 17, no. 4, pp. 265-271, 2015. 
[7] K. Gottvall and U. Waldenstrom, "Does a traumatic birth experience have an impact on future reproduction?," BJOG, vol. 109, no. 3, pp. 254-260, 2002.

[8] N. T. Mueller, R. Whyatt, L. Hoepner et al., "Prenatal exposure to antibiotics, caesarean section and risk of childhood obesity," International Journal of Obesity, vol. 39, no. 4, pp. 665-670, 2015.

[9] D. Kumar, W. Fung, R. M. Moore et al., "Proinflammatory cytokines found in amniotic fluid induce collagen remodeling, apoptosis, and biophysical weakening of cultured human fetal membranes," Biology of Reproduction, vol. 74, no. 1, pp. 29-34, 2006.

[10] J. McLaren, D. J. Taylor, and S. C. Bell, "Increased concentration of pro-matrix metalloproteinase 9 in term fetal membranes overlying the cervix before labor: implications for membrane remodeling and rupture," American Journal of Obstetrics and Gynecology, vol. 182, no. 2, pp. 409-416, 2000.

[11] S. J. Fortunato, R. Menon, C. Bryant, and S. J. Lombardi, "Programmed cell death (apoptosis) as a possible pathway to metalloproteinase activation and fetal membrane degradation in premature rupture of membranes," American Journal of Obstetrics and Gynecology, vol. 182, no. 6, pp. 1468-1476, 2000.

[12] C. Hernandez-Guerrero, F. Monzon-Bordonaba, L. JimenezZamudio et al., "In-vitro secretion of proinflammatory cytokines by human amniochorion carrying hyper-responsive gene polymorphisms of tumour necrosis factor- $\alpha$ and interleukin-1 $\beta$," Molecular Human Reproduction, vol. 9, no. 10, pp. 625-629, 2003.

[13] T. Fujimoto, S. Parry, M. Urbanek et al., "A single nucleotide polymorphism in the matrix metalloproteinase-1 (MMP-1) promoter influences amnion cell MMP-1 expression and risk for preterm premature rupture of the fetal membranes," The Journal of Biological Chemistry, vol. 277, no. 8, pp. 62966302, 2002.

[14] N. H. Simhan and T. P. Canavan, "Preterm premature rupture of membranes: diagnosis, evaluation and management strategies," BJOG, vol. 112, Supplement 1, pp. 32-37, 2005.

[15] ACOG Committee on Practice Bulletins-Obstetrics, "ACOG practice bulletin no. 80: premature rupture of membranes. Clinical management guidelines for obstetrician-gynecologists," Obstetrics \& Gynecology, vol. 109, pp. 1007-1019, 2007.

[16] National Institute for Health and Care Excellence, NICE Guideline: Preterm Labour and Birth, 2015, https://www.nice .org.uk/guidance/ng25/resources/preterm-labour-and-birth1837333576645 .

[17] Y. J. Heng, S. Liong, M. Permezel, G. E. Rice, M. K. Di Quinzio, and H. M. Georgiou, "Human cervicovaginal fluid biomarkers to predict term and preterm labor," Frontiers in Physiology, vol. 6 , p. 151, 2015.

[18] A. El-Messidi and A. Cameron, "Diagnosis of premature rupture of membranes: inspiration from the past and insights for the future," Journal of Obstetrics and Gynaecology Canada, vol. 32, no. 6, pp. 561-569, 2010.

[19] J. Pinto, A. S. Barros, M. R. M. Domingues et al., "Following healthy pregnancy by NMR metabolomics of plasma and correlation to urine," Journal of Proteome Research, vol. 14, no. 2, pp. 1263-1274, 2015.

[20] V. Fanos, L. Atzori, K. Makarenko, G. B. Melis, and E. Ferrazzi, "Metabolomics application in maternal-fetal medicine," BioMed Research International, vol. 2013, Article ID 720514, 9 pages, 2013.
[21] A. Noto, V. Fanos, and A. Dessì, "Metabolomics in newborns," Advances in Clinical Chemistry, vol. 74, pp. 35-61, 2016.

[22] G. Graca, I. F. Duarte, A. S. Barros et al., "Impact of prenatal disorders on the metabolic profile of second trimester amniotic fluid: a nuclear magnetic resonance metabonomic study," Journal of Proteome Research, vol. 9, no. 11, pp. 6016-6024, 2010.

[23] A. Noto, G. Pomero, M. Mussap et al., "Urinary gas chromatography mass spectrometry metabolomics in asphyxiated newborns undergoing hypothermia: from the birth to the first month of life," Annals of Translational Medicine, vol. 4, no. 21, pp. 417-430, 2016.

[24] S. A. Julious, "Sample size of 12 per group rule of thumb for a pilot study," Pharmaceuticals Statistics, vol. 4, pp. 287-291, 2005.

[25] J. Xia, I. V. Sinelnikov, B. Han, and D. S. Wishart, "MetaboAnalyst 3.0-making metabolomics more meaningful," Nucleic Acids Research, vol. 43, no. W1, pp. W251-W257, 2015.

[26] T. R. Moore, "Amniotic fluid dynamics reflect fetal and maternal health and disease," Obstetrics \& Gynecology, vol. 116, no. 3, pp. 759-765, 2010.

[27] J. Díaz-Castro, J. Florido, N. Kajarabille et al., "A new approach to oxidative stress and inflammatory signaling during labour in healthy mothers and neonates," Oxidative Medicine and Cellular Longevity, vol. 2015, Article ID 178536, 8 pages, 2015.

[28] G. J. Dutton, Glucuronic Acid Free and Combined: Chemistry, Biochemistry, Pharmacology, and Medicine, Academic Press Inc., New York, New York, USA, 1966.

[29] D. D. M. Wayner, G. W. Burton, K. U. Ingold, L. R. C. Barclay, and S. J. Locke, "The relative contributions of vitamin E, urate, ascorbate and proteins to the total peroxyl radical-trapping antioxidant activity of human blood plasma," Biochimica et Biophysica Acta (BBA) - General Subjects, vol. 924, no. 3, pp. 408-419, 1987.

[30] P. Caboni, A. Meloni, M. Lussu et al., "Urinary metabolomics of pregnant women at term: a combined GC/MS and NMR approach," The Journal of Maternal-Fetal \& Neonatal Medicine, vol. 27, Supplement 2, pp. 4-12, 2014. 


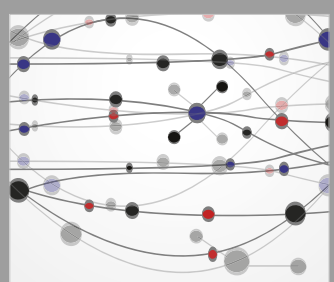

The Scientific World Journal
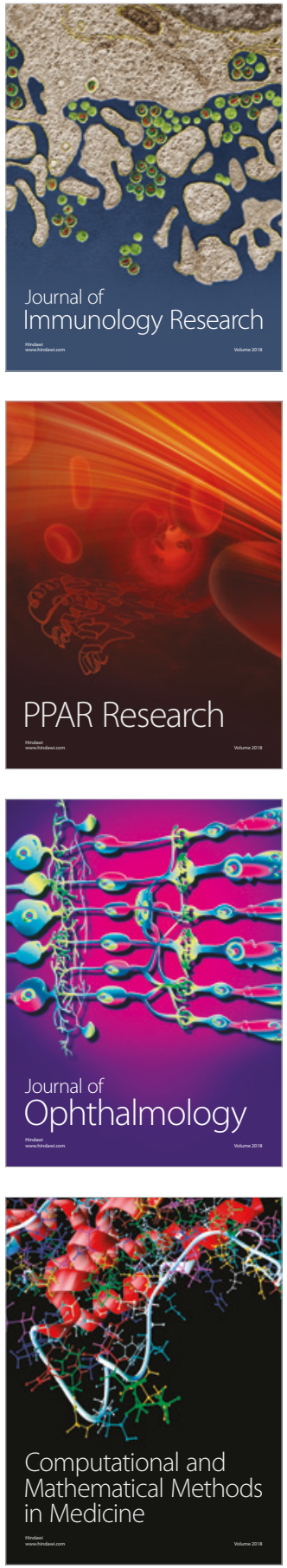

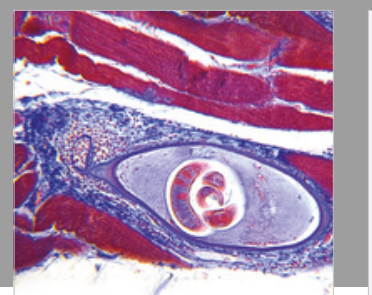

Gastroenterology Research and Practice

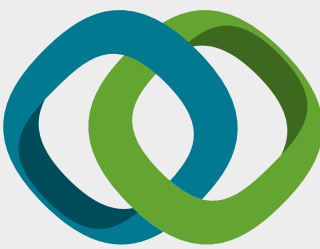

\section{Hindawi}

Submit your manuscripts at

www.hindawi.com
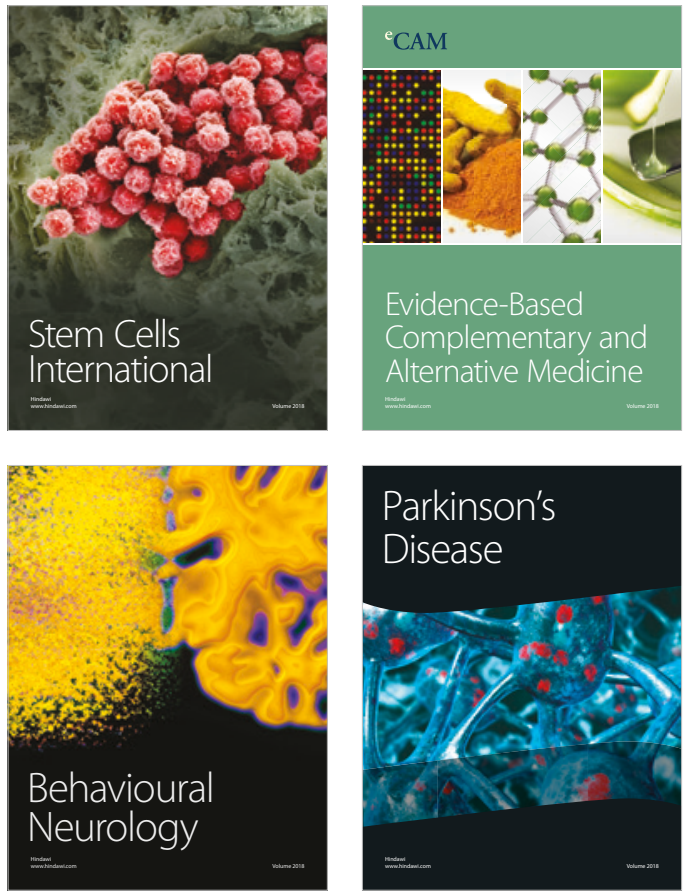

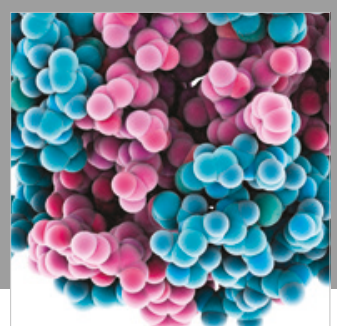

ournal of

Diabetes Research

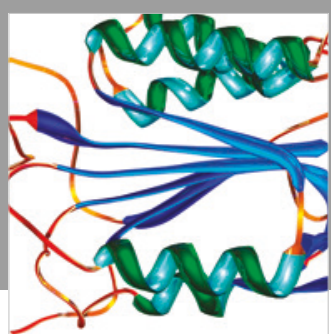

Disease Markers
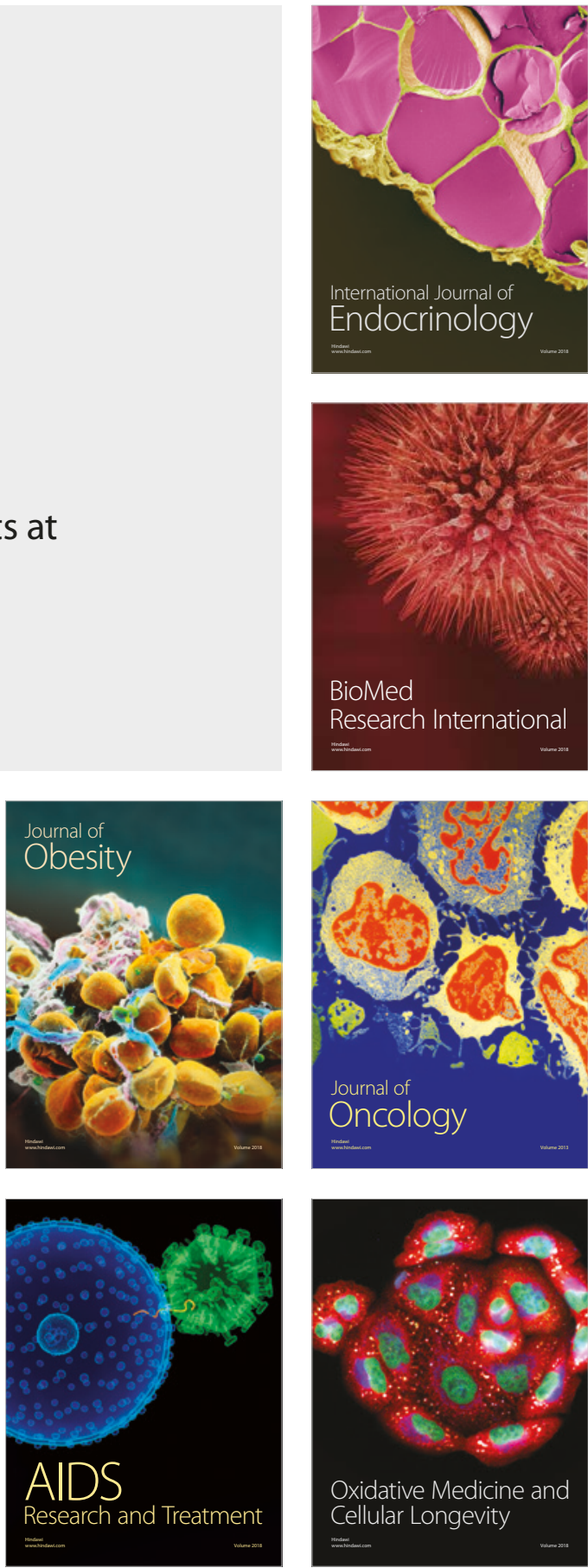